\title{
The Pathophysiology of Cholangiocarcinoma, a Review about Possible Involved Risk Factors
}

\author{
Yacob Habboush ${ }^{1 *}$ and Noor Daoud PAC ${ }^{2}$ \\ ${ }^{1}$ Orange Park Medical Center, Jacksonville, FL, USA \\ ${ }^{2}$ Baptist Health, Jacksonville, FL, USA
}

Received Date: September 05, 2018; Published Date: October 01, 2018

*Corresponding author: Yacob Habboush, Orange Park Medical Center, Jacksonville, FL, USA

\begin{abstract}
Cholangiocarcinoma (CCA) is the second most common primary hepatic malignancy. CCA is divided into three main categories, Intrahepatic CCA (iCCA), perihilar CCA (pCCA), and distal extrahepatic CCA (eCCA). iCCA is considered to be the most aggressive among all types of CCA Although the incidence of iCCA are on the rise worldwide, few of the risk factors related to iCCA have been elucidated and many have not been well-recognized. Therefore, an organized categorization of the risk factors is necessary to better understand the causes of iCCA. Consequently, this would help in screening those individuals that are at a higher risk for iCCA. We have proposed a categorization of risk factors based on previously published literature in the following manner: well-established risk factors, emerging risk factors, suspected risk factors, and unknown risk factors. Hence, will help medical and public health professionals in identifying determinants of health that might be highly influential and be able to prevent a disease with a poor prognosis.
\end{abstract}

Keywords: Hepatocellular carcinoma, cholangiocarcinoma, intrahepatic cholangiocarcinoma, risk factors

Abbreviations: CCA: Cholangiocarcinoma; iCCA: Intrahepatic CCA; pCCA: perihilar CCA; eCCA: distal extrahepatic CCA; HCC: hepatocellular carcinoma; HBV: Chronic Hepatitis B; HCV: Hepatitis C

\section{Introduction}

Liver solid lesions can commonly be divided into two categories, as benign and malignant liver lesion. Common benign liver lesions include, hepatic hemangioma, focal nodular hyperplasia, and hepatic adenoma. On the other hand, the common malignant hepatic lesions include hepatocellular carcinoma (HCC), cholangiocarcinoma (CCA), and other metastatic diseases. CCA encompasses cancer tissue that arises from the epithelial cells in the intrahepatic or extrahepatic biliary tree. CCA is the second most common primary hepatic cancer following HCC [1,2]. CCA arises from the epithelial cells of the intrahepatic and extrahepatic bile ducts. The cause of the abnormal proliferation in the bile ducts' epithelial cells leading to CCA are unknown. CCA is divided into three main categories which are Intrahepatic CCA (iCCA), Perihilar CCA (pCCA) and distal extrahepatic CCA (eCCA). The more common type of CCA is the pCCA with an estimate of $50-60 \%$ of all CCA which usually involves the bifurcation of the bile ducts, while the least common type of CCA is the iCCA, which involves the liver parenchyma, with a ratio of $10-20 \%$ of all CCA.

eCCA makes around 20\% of all the CCA. The remaining 5\% of CCA are found to be multifocal [3,4]. iCCA is recognized to be one of the highly aggressive tumors in the liver with poor prognoses [5]. Incidences have been on the rise for the past four decades, especially in the United States of America, with an increase of $128 \%$ in incidence. However, the other subtypes of CCA seem to maintain minimal fluctuant incidence with a rise of only $5 \%$ throughout the same period of time [6]. Due to the rise in iCCA incidence and the pronounced poor prognosis, a better understanding of the risk factors for iCCA is needed. In this article, we essentially focus on the risk factors leading to iCCA in the following proposed categorization of well-established risk factors, emerging risk factors, suspected risk factors, and unknown risk factors.

\section{Risk factors:}

\section{Well-established risk factors}

These risk factors will lead to an inflammatory pathway and progress to a chronic biliary inflammation with an increased cellular turnover. The chronic inflammatory response will orchestrate the tissue repair process which will lead to cellular turnover with an increased association between DNA damage and cancer development. Figure 1 demonstrates the chronic inflamation cycle. The following are the well-established risk factors for iCCA.

i. Flukes: Liver flukes infestation is highly common in Southeast Asia especially in Thailand, where there is the highest incidence of iCCA with a strong correlation to the liver fluke 
Opisthorchis viverrini (OV) [7]. Clonorchis sinensis is another liver fluke known for its correlation with iCCA which is also found in the southeast region of Asia but more prevalent in China $[8,9]$. Parasitic infections will lead to a chronic inflammation in the epithelial layer of the biliary tree, this inflammation process might not only lead to iCCA but it is also a risk factor for other non-malignant hepatobiliary diseases, such as cholangitis, and cholangiolithiasis which in turn will increase the risk for iCCA [10]. Liver flukes infestation increases the risk for iCCA with an odds ratio of 3.55 (95\% Cl: 1.60-7.89), $\mathrm{P}=0.002$ [11].

ii. Liver Cysts: Fibropolycystic liver disease and more specifically, choledochal cystic disease (Caroli's disease), is a rare congenital malformation of the bile ducts which results in segmental dilation of the large intrahepatic bile ducts which is more common in patients with Asian descent [12,13], the dilated segments are at high risk of chronic inflammation and consequently an extensive fibrosis deposition around the biliary tree which will lead to bile steatosis, cholangiolithiasis, and ascending cholangitis [14]. Although Caroli's disease is rare, it has been a well-documented risk factor for iCCA [1]. Fibropolycystic liver disease increases the risk for iCCA by $15 \%$ after the second decade with an average age of 34 years. If this cystic disease is left untreated then the overall incidence of malignancy is $28 \%$ [7].

iii. Hepatolithiasis: Hepatolithiasis, which is also referred to as recurrent pyogenic cholangitis, is the impaction of intrahepatic stones in the intrahepatic biliary tree leading to chronic inflammation that is more common in the Eastern area of Asia [15]. Incidence of hepatolithiasis in this region range from $2 \%$ to $25 \%$, while incidence in the western countries range from $0.6 \%$ to $1.3 \%$ [16] the correlation between iCCA and hepatolithiasis is well established with an odds ratio that is nearly twice more than those patients with only choledocholithiasis, the odds ratio for choledocholithiasis alone is 11.79 (95\%C: 4.17-33.35) while odds ratio with choledocholithiasis and hepatolithiasis is 22.40 (95\%Cl: 8.28-60.58) [17].

iv. Thorotrast: Thorotrast is a combination of thorium dioxide and dextrin, which is a radioactive substance used in the 1950s for x-rays as a contrast agent, it was later found to be toxic and halted its use [18]. Intravascularly injected thorotrast could stay in the blood for life and over $60 \%$ of its components mainly deposit in the liver, this may lead to chronic inflammation and damage to the liver [19]. In a sample of iCCA, microsatellite instability was noted to be $62.5 \%$ inpatient that have been exposed to thorotrast, on the other hand, patients with no history of thorotrast exposure, the microsatellites instability is lower at only $22.7 \%$ [20]. Thorotrast is associated with multiple malignancies through multiple genomic mutations, the key genes affected in thorotrast induced iCCA are the K-ras and p53 mutations which were both found to be higher in patients with thorotrast deposition in the liver than patients with non-thorotrast induced iCCA [21].

v. Primary Sclerosing Cholangitis : Primary Sclerosing Cholangitis (PSC) is a chronic inflammation of the biliary tree that leads to fibrosis and occlusion of the intra and extrahepatic bile ducts, this inflammatory process will increases the risk for iCCA by $9-20 \%$ [22]. The risk of iCCA increases by $0.5 \%$ to $1.5 \%$ each year after establishing the diagnosis with PSC [23]. Up to $50 \%$ of iCCA are diagnosed concurrently with PSC or within one year of PSC diagnosis, which imposes the necessity for regular surveillance and early diagnoses of PSC [24,25].

\section{Emerging risk factors}

Pathways and progression: The following risk factors initiate a process of chronic regenerative stimulus which will then stimulate stem cells to differentiate into cancerous cells such as HCC and CCA. This mechanism of cell progression and differentiation supports the documented similar association of risk factor between both of HCC and CCA. These risk factors are:

i. Cirrhosis: Cirrhosis is a well-established risk factor for HCC; however, its association with iCCA has not been well recognized, a recent meta-analysis has shown that cirrhosis is, in fact, a risk factor for iCCA with an overall odds ratio of 22.9 (95\% CI: 18.2-26.8) [26-27]. This correlation was not fully acknowledged at the beginning of the 20th century as Cirrhosis was not thought to be related to CCA [28]. Conversely, other studies have shown that around $24 \%$ of patients with iCCA have a concurrent diagnosis of cirrhosis [29]. In the chronic cases of cirrhosis, iCCA might be left undiagnosed or misdiagnosed as HCC due to the chronic inflammation of the liver which makes screening and diagnosing patients with iCCA challenging [30].

ii. Chronic Hepatitis B and Hepatitis C : Chronic Hepatitis B (HBV) and Hepatitis C (HCV) infections of the liver could lead to an acute disease or more commonly a chronic inflammation of the liver. The association of these viral infections with iCCA is recognized, however, the prevalence and risk estimate of HCV in comparison to HBV is controversial, as to which one holds the worst prognosis with iCCA [31]. The prevalence of HCV is more common than HBV in iCCA with an estimated prevalence of $20 \%$ and $10 \%$ respectively [31]. Pooled risk estimate of iCCA in HCV was found to be higher than HBV with an odds ratio of 3.38 (95\% CI: 2.72 to 4.21) [32]. In another meta-analysis study it showed that the prevalence of HBV (38\%) is more common in iCCA than HCV (13\%) with an overall odds ratio of $5.10(95 \% \mathrm{Cl}$ : 2.91-8.95) and 4.84 (95\% Cl: 2.41-9.71) respectively [26]. A more recent meta-analysis showed that overall survival is shortened in patients with iCCA due to HCV in comparison to HBV with a pooled hazards ratio of 2.64 (95\% CI: 1.77-3.93), however, HBV was associated with a higher rate of cirrhosis (odds ratio=6.44, 95\% CI: 4.33-9.56) [33].

iii. Obesity: Obesity is considered to be one of the most problematic health concerns that are on the rise particularly in the western countries such as the United States of America, where the estimated obese (body mass index $>30$ ) population is around $35 \%$ of the total adults 20 years of age or older $[34,35]$ obesity is considered to be a risk factor for multiple malignancies including CCA, however, its association with iCCA is not well 
established [34]. A study has suggested that obesity is related to iCCA more than other types of CCA $(p=0.01)$ [36]. Another study has demonstrated that the risk factor of obesity for iCCA has an odds ratio of $1.56(95 \% \mathrm{Cl}$ : $1.26-1.94)$ [26]. On the other hand, further studies have shown no relationship between obesity and iCCA $[37,38]$.

iv. Diabetes Mellitus: Diabetes Mellitus (DM) is characterized by hyperglycemia in affected patients. World Health Organization has modulated an estimation that demonstrates DM to be the seventh global leading cause of death in 2030 [39]. The association of DM and iCCA is debatable. Two large independent meta-analyses have shown a positive correlation with all types of CCA and the strongest association was with iCCA (Overall Relative Risk=1.97; 95\% CI: 1.57-2.46; P=0.025) [40] and (Overall Odds Ratio 1.89; 95\% Cl: 1.74-2.07) [26]. Nevertheless, other three case-control studies have advocated no relationship between DM and iCCA, all of wish did not show a significant difference in DM prevalence between the study and control group (Estimated Risk= 1.5 (95\% Cl: 0.6-3.8) [41], Estimated Risk=1.95 (95\%Cl: 0.6-5.8) [42], and Estimated Risk=1.43 (95\% Cl: 0.8-2.6) [36].

v. Alcohol: Alcohol may lead to liver parenchyma damage through the process of oxidative stress [43]. Assessing alcohol consumption as a risk factor for iCCA has yielded inconsistent results [44]. A meta-analysis of 11 independent studies has determined an association between alcohol and iCCA with an overall Odds Ratio of 2.81 (95\% CI: 1.52-5.21) [26]. More than three alcoholic beverages per week were related to higher incidence of all types of CCA (Odds Ratio=2.13; 95\%Cl: 1.00-4.55) [45] Whereas other case-control studies have failed to link alcohol to iCCA [41].

vi. Non-alcoholic steatohepatitis (NASH): NASH is a welldocumented risk factor for HCC, however, its association with iCCA has not been well identified in the past. More recent studies have shown a possible correlation between NASH and iCCA. A case study has shown that NASH is an independent risk factor for iCCA with a multivariate analysis and an odds ratio of $3.36(95 \% \mathrm{Cl}$ : 1.15-10.2, P-value $=0.0269$ ) [46].

\section{Suspected Risk factors}

Pathways and progression: This category of risk factors is thought to affect the DNA structure by directly damaging the integrity of the DNA replication through the DNA polymerase incorrectly matching nucleotides during DNA replications. Therefore, as the mutation occurs, the risk of malignant cells development increases. These risk factors are:

i. Occupational Factors: Occupational factors and hazards might be a critical yet preventable risk factor. CCA has been reported in workers in a printing factory in Osaka, Japan. Those patients were exposed to high volumes of inhaled 1,2-dichoropropane dichloromethane during the cleaning procedure of the printing machines. From the total 11 CCA cases, five were diagnosed with iCCA and six with eCCA. The diagnosis of CCA was established between 7 to 20 years after the first exposure to 1,2-dichoropropane dichloromethane [47]. Further statistical analysis has revealed that a cumulative exposure to 1,2-dichoropropane dichloromethane is associated with an increased Relative Risk to all types of CCA [48]. A more recent study have completed a whole-exome analysis on four of the above mentioned cases who were exposed to haloalkanes and found out that on average of 44.8 somatic mutations were detected per $\mathrm{Mb}$ in the genome of the CCA tissue of the affected workers which is 30 -fold higher than what was found in other CCA tissue in the control group [49].

ii. Inflammatory Bowel Disease: Inflammatory Bowel Disease (IBD) is a known risk factor for multiple gastrointestinal malignancies [50]. However, only a few studies have targeted IBD as a risk factor for iCCA. A meta-analysis assessing the risk of iCCA and eCCA in patients affected by IBD has reported a pooled Relative Risk for iCCA of 2.61 (95\% Cl: 1.72-3.95) while eCCA had pooled Relative Risk of 1.47 (95\%Cl: 1.10-1.97) [51].

iii. Helicobacter Species: Helicobacter species (H.sp.) have been linked to multiple gastrointestinal malignancies; however, its relationship to CCA is not well understood. A recent study has suggested a possible link between all types of CCA and H.sp., with higher prevalence in eCCA and common bile duct cancer rather than iCCA [52].

\section{Unknown Risk factors}

These factors are not yet identified and might not be well correlated as a risk factors, however, they do pertain to be present in some of the case reports mentioned in the literature. These risk factors are:

i. Genetic polymorphisms: Although the incidence of iCCA are on the rise worldwide, the lack of appropriate genomic platform identification that is susceptible for mutation which would increase the risk for iCCA is not well- documented [53]. Somatic mutations in KRAS, TP53, APC, DKN2A, ROB01, ROBO2, RUNX3, and SMAD4 were found to be associated with iCCA [54]. Additional study has revealed an upregulation is PDZK1IP1, EEF1A2 and RPL41 genes in iCCA patients [55]. Demographic criteria might also be an influential risk fact, as per US Surveillance, Epidemiology and End Results (SEER) data; the prevalence of iCCA is highest in Hispanic Americans and Native Americans with the greatest mortality increase in Hispanics and females in comparison to males [56].

ii. Environmental Factors: iCCA prevalence has not been well assessed in relation to the different environmental exposures, however, a link might be present [10]. A case-control study examined patients with chronic asbestos exposure of over 30 years and found out that there is a higher risk of developing CCA. iCCA risk was higher than eCCA as the adjusted Odds Ratio was $4.81,95 \% \mathrm{Cl}: 1.73-13.33$ and $2.09,95 \% \mathrm{Cl}$ : $0.83-5.27$ respectively [57].

iii. Smoking: Smoking has been associated with a vast majority of known malignancies, however, its correlation to iCCA 


\section{Novel Approaches in Drug Designing \& Development}

is not fully understood. A meta-analysis has found an insignificant correlation between smoking and iCCA (overall Odds Ratio= 1.31 (95\%Cl: 0.95-1.82) [26]. Although other studies have shown a clear correlation (adjusted Odds Ratio=1.6; 95\% CI: 1.3-2.1; $\mathrm{P}=<$ 0.001 ) and other case-control study have establish a parallel result (Odds Ratio=1.931, 95\% CI: 1.00-3.73) [38,58].

iv. Strategies for early detection or prevention of iCCA: The principle key factor to manage the increasing incidence of iCCA worldwide is primary prevention, which would translate into risk factors prevention. Early detection is another significant component in management to halt the progression of iCCA and any further complications. There are some general protective factors that could reduce the overall incidence and prevalence of iCCA such as aspirin and metformin with an adjusted odds ratio of 0.35 (95\% Cl: $0.29-0.42 ; \mathrm{P}=<0.001)$ and $0.4(95 \% \mathrm{Cl}: 0.2-0.9$; $\mathrm{P}=0.04$ ) respectively $[38,59]$. A less profound protective effect is the use of statins as a case-control study has shown that statins reduce the risk of CCA which is also dose related, adjusted odds ratio $=0.80,95 \% \mathrm{Cl}: 0.71-0.90$ [60]. Table 1 shows more specific protective factors a Figure 1 gainst iCCA.

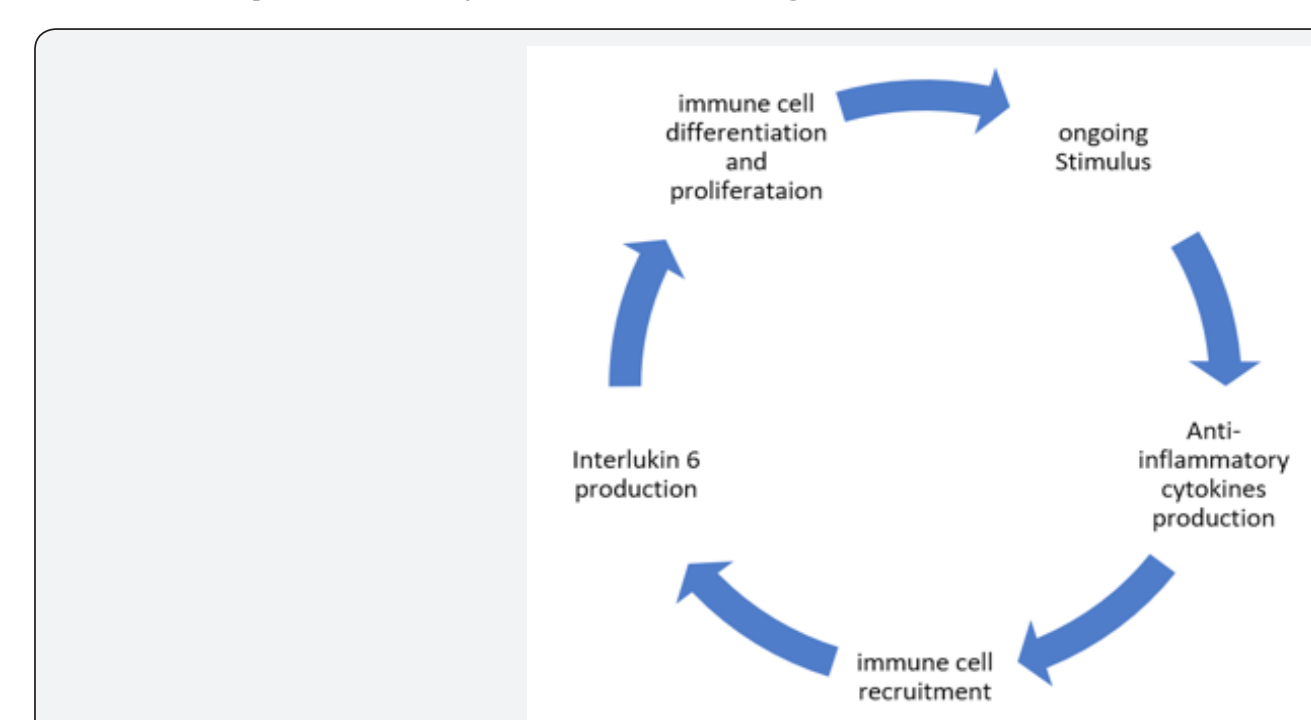

Figure 1: Chronic Inflammation Cycle.

Table 1: Specific protective effects against risk factors for iCCA.

\begin{tabular}{|c|c|c|}
\hline \multirow{4}{*}{$\begin{array}{c}\text { Pathways and } \\
\text { Progression }\end{array}$} & Risk Factors & Protective Factors \\
\hline \multirow{4}{*}{ Chronic inflammation } & Liver flukes & Public health awareness \\
\cline { 2 - 3 } & Liver cysts & Family history and Public health \\
\cline { 2 - 3 } & Hepatolithiasis & Ductal dilation and stents \\
\cline { 2 - 3 } & Thorotrast & Screening \\
\hline \multirow{4}{*}{ Chronic regeneration } & Primary sclerosing cholangitis & Screening \\
\cline { 2 - 3 } & Cirrhosis & Immunization \\
\cline { 2 - 3 } & Chronic hepatitis B & Early detection \\
\cline { 2 - 3 } & Chronic hepatitis C & Weight reduction (Diet and physical activity) \\
\cline { 2 - 3 } & Obesity & Weight reduction (Diet and physical activity) \\
\cline { 2 - 3 } & Diabetes mellitus & Alcohol cessation \\
\cline { 2 - 3 } & Alcohol & Weight reduction (Diet and physical activity) \\
\hline \multirow{3}{*}{ Direct DNA damage } & NASH & Occupational health \\
\cline { 2 - 3 } & Occupational factors & Smoking cessation and Dietary manipulation \\
\cline { 2 - 3 } & Inflammatory bowel disease & Antibiotics \\
\cline { 2 - 3 } & Helicobacter species & Genetic counseling \\
\hline \multirow{3}{*}{ Unknown } & Genetic polymorphisms & Public health initiatives \\
\hline
\end{tabular}




\section{Novel Approaches in Drug Designing \& Development}

\section{Conclusion}

Understanding the risk factors for iCCA is essential to identify and screen those with high risk. Although current knowledge is limited, specifically in the genetic pathway that leads to the development to iCCA with no sensitive molecular marker, it is essential to categorize risk factors to assess, anticipate, and screen patients with the appropriate resources. The four suggested categories are the well-established, emerging, suspected, and unknown risk factors. Further research should focus on establishing a stronger link between iCCA and the emerging, suspected, and unknown risk factors to move them into the well establish category to enable medical and public health professionals to focus on prevention through managing determinants of health.

\section{References}

1. Plentz RR, NP Malek (2015) Clinical presentation, risk factors and staging systems of cholangiocarcinoma. Best Pract Res Clin Gastroenterol 29(2): 245-252.

2. Zhang H, Yang $T$, Wu M, Shen F (2016) Intrahepatic cholangiocarcinoma: Epidemiology, risk factors, diagnosis and surgical management. Cancer Lett 379(2): 198-205

3. Khan SA, Toledano MB, Taylor Robinson SD (2008) Epidemiology, risk factors, and pathogenesis of cholangiocarcinoma. HPB 10(2): 77-82.

4. Ettel M, Eze O, Xu R (2015) Clinical and biological significance of precursor lesions of intrahepatic cholangiocarcinoma. World J Hepatol $7(25): 2563-2570$.

5. Sia D, Tovar V, Moeini A, Llovet JM (2013) Intrahepatic cholangiocarcinoma: pathogenesis and rationale for molecular therapies. Oncogene 32(41): 4861-4870.

6. Saha SK, Zhu AX, Fuchs CS, Brooks GA (2016) Forty-Year Trends in Cholangiocarcinoma Incidence in the U.S.: Intrahepatic Disease on the Rise. Oncologist 21(5): 594-599.

7. Khan, Thomas HC, Davidson BR, Taylor Robinson SD (2005) Cholangiocarcinoma. Lancet 366(9493): 1303-1314.

8. Sithithaworn, Puangrat Yongvanit, Kunyarat Duenngai, Nadda Kiatsopit, Chawalit Pairojkul (2014) Roles of liver fluke infection as risk factor for cholangiocarcinoma. Journal of Hepato Biliary-Pancreatic Sciences 21(5): 301-308.

9. Lee SU, S Huh (2004) Variation of nuclear and mitochondrial DNAs in Korean and Chinese isolates of Clonorchis sinensis. Korean J Parasitol 42(3): 145-148.

10. Al Bahrani R, Yasser Abuetabh, Nikolas Zeitouni, Consolato Sergi (2013) Cholangiocarcinoma: risk factors, environmental influences and oncogenesis. Annals of Clinical \& Laboratory Science 43(2): 195210.

11. Peng NF, Yang YF, Su ZX, Chen B, Su M, Qi LN, et al. (2011) Evaluation of risk factors and clinicopathologic features for intrahepatic cholangiocarcinoma in Southern China: a possible role of hepatitis B virus. Ann Surg Oncol 18(5): 1258-1266.

12. Gupta AK, Gupta A, Bhardwaj VK, Chansoria M (2006) Caroli's disease. Indian J Pediatr 73(3): 233-235.

13. Desmet VJ (1992) Congenital diseases of intrahepatic bile ducts: variations on the theme "ductal plate malformation". Hepatology 16(4): 1069-1083.

14. Jang MH, YJ Lee, Kim H (2014) Intrahepatic cholangiocarcinoma arising in Caroli's disease. Clin Mol Hepatol 20(4): 402-425.
15. Mori T, Sugiyama M, Atomi Y (2006) Gallstone disease: Management of intrahepatic stones. Best Pract Res Clin Gastroenterol 20(6): 11171137.

16. Kim HJ, Kim JS, Joo MK, Lee BJ, Kim JH, et al. (2015) Hepatolithiasis and intrahepatic cholangiocarcinoma: A review. World Journal of Gastroenterology 21(48): 13418-13431.

17. Cai H, Kong WT, Chen CB, Shi GM, Huang C, et al. (2015) Cholelithiasis and the risk of intrahepatic cholangiocarcinoma: a meta-analysis of observational studies. BMC Cancer 15: 831.

18. Zhu AX, Lauwers GY, Tanabe KK (2004) Cholangiocarcinoma in association with Thorotrast exposure. J Hepatobiliary Pancreat Surg 11(6): 430-433.

19. Mori T, Kido C, Fukutomi K, Kato Y, Hatakeyama S, et al. (1999) Summary of entire Japanese thorotrast follow-up study: updated 1998 Radiat Res 152(6 Suppl): S84-S87.

20. Liu D, Momoi H, Li L, Ishikawa Y, Fukumoto M (2002) Microsatellite instability in thorotrast-induced human intrahepatic cholangiocarcinoma. Int J Cancer 102(4): 366-371.

21. Lipshutz GS, Brennan TV, Warren RS (2002) Thorotrast-induced liver neoplasia: a collective review. J Am Coll Surg 195(5): 713-718.

22. Timmer MR, Beuers U, Fockens P, Ponsioen CY, Rauws EA, et al. (2013) Genetic and epigenetic abnormalities in primary sclerosing cholangitis-associated cholangiocarcinoma. Inflamm Bowel Dis 19(8): 1789-1797.

23. Fevery J, Verslype C, Lai G, Aerts R, Van Steenbergen W (2007) Incidence, diagnosis, and therapy of cholangiocarcinoma in patients with primary sclerosing cholangitis. Dig Dis Sci 52(11): 3123-3135.

24. Bergquist A, Evon Seth (2015) Epidemiology of cholangiocarcinoma. Best Practice \& Research in Clinical Gastroenterology 29(2): 221-232.

25. Morris Stiff G, Bhati C, Olliff S, Hübscher S, Gunson B, et al. (2008) Cholangiocarcinoma complicating primary sclerosing cholangitis: a 24-year experience. Dig Surg 25(2): 126-132.

26. Palmer WC, Patel T (2012) Are common factors involved in the pathogenesis of primary liver cancers? A meta-analysis of risk factors for intrahepatic cholangiocarcinoma. J Hepatol 57(1): 69-76.

27. Michelotti GA, Machado MV, Diehl AM (2013) NAFLD, NASH and liver cancer. Nature Reviews Gastroenterology \& Hepatology 10(11): 656665.

28. Nomoto K, Tsuneyama K, Cheng C, Takahashi H, Hori R, (2006) et al. Intrahepatic cholangiocarcinoma arising in cirrhotic liver frequently expressed p63-positive basal/stem-cell phenotype. Pathol Res Pract 202(2): 71-76.

29. Shaib YH, El Serag HB, Nooka AK, Thomas M, Brown TD, et al. (2007) Risk factors for intrahepatic and extrahepatic cholangiocarcinoma: a hospital-based case-control study. Am J Gastroenterol 102(5): 10161021.

30. Vallin M, Sturm N, Lamblin G, Guillaud O, Hilleret MN et al. (2013) Unrecognized intrahepatic cholangiocarcinoma: an analysis of 993 adult cirrhotic liver explants. Clinical Transplantation 27(3): 403-409.

31. Matsumoto K, Onoyama T, Kawata S, Takeda Y, Harada K, Ikebuchi Y et al. (2015) Hepatitis B and C virus infection is a risk factor for the development of cholangiocarcinoma. Intern Med 53(7): 651-654.

32. Li H (2015) Hepatitis C virus infection and the risk of intrahepatic cholangiocarcinoma and extrahepatic cholangiocarcinoma: evidence from a systematic review and meta-analysis of 16 case-control studies. World Journal of Surgical Oncology 13: 161.

33. Wang Z, Sheng YY, Dong QZ, Qin LX (2016) Hepatitis B virus and hepatitis $\mathrm{C}$ virus play different prognostic roles in intrahepatic cholangiocarcinoma: A meta-analysis. World J Gastroenterol 22(10): 3038-3051. 
34. Parsi MA (2013) Obesity and cholangiocarcinoma. World J Gastroenterol 19(4): 457-462.

35. Ogden CL, Carroll MD, Kit BK, Flegal KM (2013) Prevalence of obesity among adults: United States, 2011-2012. NCHS Data Brief (131): 1-8.

36. Welzel TM, Graubard BI, El Serag HB, Shaib YH, Hsing AW, et al. (2007) Risk factors for intrahepatic and extrahepatic cholangiocarcinoma in the United States: a population-based case-control study. Clin Gastroenterol Hepatol 5(10): 1221-1228.

37. Welzel TM, Mellemkjaer L, Gloria G, Sakoda LC, Hsing AW, et al. (2007) Risk factors for intrahepatic cholangiocarcinoma in a low-risk population: a nationwide case-control study. Int J Cancer 120(3): 638641.

38. Chaiteerakij R, Yang JD, Harmsen WS, Slettedahl SW, Mettler TA, et al. (2013) Risk factors for intrahepatic cholangiocarcinoma: association between metformin use and reduced cancer risk. Hepatology 57(2): 648-655.

39. SaengboonmeC, SeubwaiW, Wongkham C,WongkhamS(2015) Diabetes mellitus: Possible risk and promoting factors of cholangiocarcinoma: Association of diabetes mellitus and cholangiocarcinoma. Cancer Epidemiol 39(3): 274-278.

40. Jing W, Jin G, Zhou X, Zhou Y, Zhang Y et al. (2012) Diabetes mellitus and increased risk of cholangiocarcinoma: a meta-analysis. Eur J Cancer Prev 21(1): 24-31.

41. Zhou YM (2008) Risk factors for intrahepatic cholangiocarcinoma: a case-control study in China. World Journal of Gastroenterology 14(4): 632-635.

42. Yamamoto S, Kubo S, Hai S, Uenishi T, Yamamoto T, et al. (2004) Hepatitis $\mathrm{C}$ virus infection as a likely etiology of intrahepatic cholangiocarcinoma. Cancer Sci 95(7): 592-595.

43. Aishima S, Fujita N, Mano Y, Iguchi T, Taketomi A, et al. (2013) p62+ Hyaline inclusions in intrahepatic cholangiocarcinoma associated with viral hepatitis or alcoholic liver disease. Am J Clin Pathol 134(3): 457 465.

44. Zhou YM, Zhang XF, Wu LP, Sui CJ, Yang JM (2014) Risk factors for combined hepatocellular-cholangiocarcinoma: a hospital-based casecontrol study. World J Gastroenterol 20(35): 12615-12620.

45. Manwong M, Songserm N, Promthet S, Matsuo K (2013) Risk factors for cholangiocarcinoma in the lower part of Northeast Thailand: a hospital-based case-control study. Asian Pac J Cancer Prev 14(10): 5953-5956.

46. Kinoshita M, Kubo S, Tanaka S, Takemura S, Nishioka T, et al. (2016) The association between non-alcoholic steatohepatitis and intrahepatic cholangiocarcinoma: A hospital based case-control study. J Surg Oncol 113(7): 779-783.

47. Kumagai S, Kurumatani N, Arimoto A, Ichihara G (2013) Cholangiocarcinoma among offset colour proof-printing workers exposed to 1,2-dichloropropane and/or dichloromethane. Occup Environ Med 70(7): 508-510.
48. Kumagai S, Sobue T, Makiuchi T, Kubo S, Uehara S, et al. (2016) Relationship between cumulative exposure to 1,2-dichloropropane and incidence risk of cholangiocarcinoma among offset printing workers. Occup Environ Med 73(8): 545-552.

49. Mimaki S, Totsuka Y, Suzuki Y, Nakai C, Goto M, et al. (2016) Hypermutation and unique mutational signatures of occupational cholangiocarcinoma in printing workers exposed to haloalkanes. Carcinogenesis 37(8): 817-826.

50. Axelrad JE, Lichtiger S, Yajnik V (2016) Inflammatory bowel disease and cancer: The role of inflammation, immunosuppression, and cancer treatment. World J Gastroenterol 22(20): 4794-4801.

51. Huai JP, Ding J, Ye XH, Chen YP (2014) Inflammatory bowel disease and risk of cholangiocarcinoma: evidence from a meta-analysis of population-based studies. Asian Pacific Journal of Cancer Prevention 15(8): 3477-3482.

52. Kaewpitoon SJ, Loyd RA, Rujirakul R, Panpimanmas S, Matrakool L, et al. (2016) Helicobacter Species are Possible Risk Factors of Cholangiocarcinoma. Asian Pac J Cancer Prev 17(1): 37-44.

53. Xie D, Ren Z, Fan J, Gao Q (2016) Genetic profiling of intrahepatic cholangiocarcinoma and its clinical implication in targeted therapy. Am J Cancer Res 6(3): 577-586.

54. Kim YH, Hong EK, Kong SY, Han SS, Kim SH (2016) Two classes of intrahepatic cholangiocarcinoma defined by relative abundance of mutations and copy number alterations. Oncotarget 7(17): 2382523836.

55. Yang G, Zong H (2016) Overexpression of PDZK1IP1, EEF1A2 and RPL41 genes in intrahepatic cholangiocarcinoma. Molecular Medicine Reports 13(6): 4786-4790.

56. (2013) US Surveillance, E.a.E.R. Cancer Statistics.

57. Brandi G, Di Girolamo S, Farioli A, de Rosa F, Curti S, et al. (2013) Asbestos: a hidden player behind the cholangiocarcinoma increase? Findings from a case-control analysis. Cancer Causes Control 24(5): 911-918.

58. Liu ZY, Zhou YM, Shi LH, Yin ZF (2011) Risk factors of intrahepatic cholangiocarcinoma in patients with hepatolithiasis: a case-control study. Hepatobiliary Pancreat Dis Int 10(6): 626-631.

59. Choi J, Hassan Ghoz, Thoetchai Peeraphatdit, Lewis Rowland Roberts (2016) Risk Factors for Cholangiocarcinoma: Aspirin-use and the Risk of Cholangiocarcinoma. Hepatology 64(3).

60. Peng YC, Lin CL, Hsu WY, Chang CS, Yeh HZ, et al. (2015) Statins are associated with a reduced risk of cholangiocarcinoma: a populationbased case-control study. Br J Clin Pharmacol 80(4): 755-761. 
Your next submission with Juniper Publishers will reach you the below assets

- Quality Editorial service

- Swift Peer Review

- Reprints availability

- E-prints Service

- Manuscript Podcast for convenient understanding

- Global attainment for your research

- Manuscript accessibility in different formats

( Pdf, E-pub, Full Text, Audio)

- Unceasing customer service

Track the below URL for one-step submission https://juniperpublishers.com/online-submission.php 